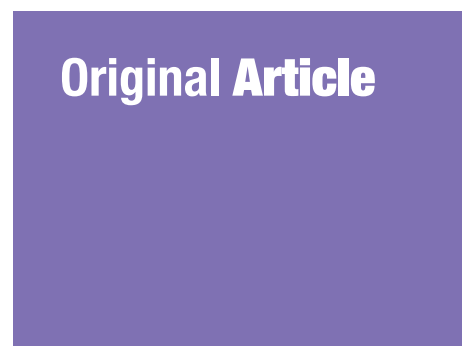

Submitted: 7 Dec 2016

Accepted: 19 Jun 2017

Online: 18 Aug 2017

\section{Effects of Whole Body Vibration on Glycemic Indices and Peripheral Blood Flow in Type II Diabetic Patients}

\author{
Nuttaset Manimmanakorn ${ }^{1}$, Apiwan Manimmanakorn ${ }^{2}$, Warinthorn \\ PhutTharak ${ }^{3}$, Michael J HamLin ${ }^{4}$
}

1 Department of Physical Medicine and Rehabilitation, Faculty of Medicine, Khon Kaen University, Khon Kaen, 40002, Thailand

2 Department of Physiology, Faculty of Medicine, Khon Kaen University, Khon Kaen, 40002, Thailand

3 Department of Radiology, Faculty of Medicine, Khon Kaen University, Khon Kaen 40002, Thailand.

4 Department of Tourism, Sport and Society, PO Box 85084 Lincoln University, Lincoln, 7647, Christchurch, New Zealand

To cite this article: Manimmanakorn N, Manimmanakorn A, Phuttharak W, Hamlin MJ. Effects of whole body vibration on glycemic indices and peripheral blood flow in type II diabetic patients. Malays J Med Sci. 2017;24(4): 55-63. https://doi.org/10.21315/mjms2017.24.4.7

To link to this article: https://doi.org/10.21315/mjms2017.24.4.7

\begin{abstract}
Background: Whole body vibration (WBV) training is a regime of training on a vibration platform that provides oscillatory movement to the body. Vibration training may be a potentially useful therapeutic strategy to control diabetes and its complications. This study aimed to evaluate the effect of WBV on glycemic indices and peripheral blood flow in type II diabetic patients.

Methods: A parallel group clinical trial was conducted with 1:1 allocation ratio at Khon Kaen University between February and May 2010. The study included diabetic patients receiving diet or oral medication control over the previous year and excluded patients with serious medical and musculoskeletal disorders. Forty type II diabetic patients [14 males, 26 females, 63.2 (7.7) y, mean (SD)] were randomised into two groups (WBV and control) by computer software using a block of four design. The WBV group was given two sets of six one-minute vibration squats, three times per week for twelve weeks. The control group maintained their normal physical activity levels. The primary outcome was the patients glycemic indices.

Results: We found no significant difference in glycosylated haemoglobin (HbA1c), fasting blood sugar, insulin level and insulin sensitivity between WBV and control groups. Compared to the control group, WBV training resulted in a substantial reduction in resting diastolic blood pressure $-7.1 \mathrm{mmHg}(95 \% \mathrm{CI}:-\mathbf{- 1 0 . 9},-\mathbf{- 3 . 3}, \boldsymbol{P}=\mathbf{0 . 0 0 1})$ and peak systolic velocity $-7.3 \mathrm{~cm} . \mathrm{sec}^{-1}(95 \% \mathrm{CI}$ : $-14.7,-0.03, P=0.049)$, but made little difference to resting heart rate, systolic blood pressure, end diastolic velocity, and popliteal artery diameter.

Conclusion: Whole body vibration improved resting diastolic blood pressure and peak systolic velocity, however, any beneficial effect of WBV on glycemic indices remains unclear.
\end{abstract}

Keywords: vibration, diabetes mellitus, blood circulation, blood pressure, clinical trial

\section{Introduction}

Type II diabetes or adult-onset diabetes is the most common type of diabetes and leads to complications including retinopathy, nephropathy, neuropathy and cardiovascular disease (1). Currently, management of diabetes consists of medication, diet control, exercise and lifestyle modification.

Whole body vibration (WBV) is an innovative exercise training regime that utilises electric motors to provide vibration movement to 
an individual standing on a platform. Vibration training has been suggested to be a useful alternative or supplement to current exercise prescriptions for diabetic patients (2). It is thought that vibration training stimulates muscle contraction, increases peripheral blood flow, and energy consumption $(3,4)$, all of which may help with diabetes and its complications.

Whole body vibration stimulates muscle contraction by activating muscle spindles, thereby enhancing stretch-reflex activation (5). Normal regular WBV training is suggested to generate muscle adaptation similar to normal resistance exercise training (6). It is well accepted that regular physical exercise increases glucose uptake in exercising muscle, resulting in enhanced insulin sensitivity in diabetics (7). For that reason, regular WBV training may help diabetic patients to control glucose metabolism by reducing fasting blood sugar (FBS), glycosylated hemoglobin (HbA1c; a marker for evaluating average blood sugar levels over the previous 3 months), fasting insulin and increasing insulin sensitivity.

Previous research indicates controversial effects of WBV on glucose metabolism in diabetes patients. Baum et al. (2) revealed FBS and HbA1c levels in type II diabetics were reduced by approximately $6.3 \%$ and $0.3 \%$ respectively after 12 weeks of WBV using a horizontal swinging platform $(30-35 \mathrm{~Hz}, 2$ $\mathrm{mm}$ amplitude, 8 bouts of 30 seconds, 3 days a week). More recently, del Pozo-Cruz et al. (9) reported 12 weeks of WBV training $(12-16 \mathrm{~Hz}, 4$ $\mathrm{mm}, 3$ days a week) significantly improved levels of FBS and HbA1c. However, in contrast to the beneficial effects reported by Baum et al. (2) and del Pozo-Cruz et al. (9), Behboudi et al. (8) found that FBS and HbA1c levels were not significantly improved after 8 weeks of WBV training (30 $\mathrm{Hz}, 2 \mathrm{~mm}, 3$ days a week). It therefore seems that the effect of WBV on glucose metabolism in diabetics remains unclear and requires further investigation.

As a consequence of a disrupted glucose metabolism, diabetics also suffer from peripheral vascular disorders. One of the purported benefits of whole body vibration training is improved vascular blood flow. However, the beneficial change in local blood flow with vibration still remains controversial. Kerschan-Schindl et al. (10) showed mean blood flow velocity in the popliteal artery of healthy adults increased $100 \%$ after 9 minutes of vibration training (26 $\mathrm{Hz}, 3 \mathrm{~mm}$,). In addition, Lohman et al. (11) revealed skin blood flow of the lower leg was increased by approximately $147 \%$ after 3 bouts of 60 seconds of vibration training $(30 \mathrm{~Hz}, 5-6$ $\mathrm{mm}$ ). Lythgo et al. (12) suggested the increased leg blood flow accompanying WBV in healthy volunteers was related to high frequency and high amplitude of vibration. However, using high frequency, Button et al. (13) and Hazell et al. (14) reported no significant difference in local blood flow in healthy participants between vibration and control groups. In diabetic patients, and in contrast to Lythgo et al. (12) findings on healthy subjects, Johnson et al. (15) showed low frequency $(26 \mathrm{~Hz})$ and low amplitude (2 $\mathrm{mm}$ ) vibration increased blood flow (to the skin area in the feet). Similarly, Sañudo et al. (16) revealed significant improvement of femoral artery blood flow after 12 weeks of WBV training in diabetic patients $(12-16 \mathrm{~Hz}, 4 \mathrm{~mm}, 3$ days a week). Whether WBV can have a positive effect on local blood flow of diabetic patients remains controversial and requires further investigation.

The aim of this study was to evaluate the effect of WBV as an additional therapy to diet or oral medication in our clinical setting on FBS, HbA1c, fasting insulin levels, insulin sensitivity and peripheral vascular blood flow on a cohort of Type II diabetic patients.

\section{Materials and Methods}

\section{Study Design}

A randomised control trial was conducted in parallel groups with an allocation ratio 1:1 at Srinagarind Hospital, KhonKaen University between February and May 2010.

\section{Study Population}

The type II diabetic patients who were diagnosed by a registered medical physician and came to the primary care unit of Srinagarind Hospital Khon Kaen University were recruited in this study. The patients were recruited through poster advertisement and primary doctors who took care of the patients. Forty patients who met the following criteria were tested pre(baseline) and 12 weeks after (post) intervention. Inclusion criteria for type II diabetics included, patients receiving diet control programs or oral medication for diabetes over the last year. Exclusion criteria included (i) having any serious medical diseases (e.g. diabetic retinopathy, myocardial infarction, uncontrolled hypertension or renal failure), (ii) having previous hip, knee or shoulder operations or, (iii) any uncontrolled back, ankle, knee and hip pain. 


\section{Randomisation and Allocation Concealment}

The participants were randomly allocated into the WBV or the control group. The randomised allocation was performed by the office of Rehabilitation Medicine Department without any influence of the investigators. The allocation sequence was generated by computer software using a block size of four, and was concealed by sequentially numbered, sealed and opaque envelopes.

\section{Blinding}

The patients could not be blinded because this study compared WBV training and nontraining. The period of training was 12 weeks which made it difficult to conceal the patient groups as all patients lived in the same village. However, the investigators who measured the physiological outcomes such as weight, height, heart rate and blood pressure and completed blood test analyses at baseline and at the end of study were blinded to the allocation treatment. In addition, the radiologist who performed the ultrasound Doppler before and after training was also blinded to patient group information.

\section{Sample Size Calculation}

The sample size estimation which compared means of two independent groups was calculated based on a type I error $(\alpha)=0.05$, type II error $(\beta)=0.2$, mean difference $\left(\mu_{1}-\mu_{2}\right)=5$, variance $=25$ from Baum et al. study (2). The number of participants required using this calculation was 16 per group. After considering a normal rate of drop outs (20 \%), the total sample size per group was 20 per group.

\section{Study Protocol}

Forty diabetic patients, 20 for WBV and 20 for control group, were included in this study. Three people in the WBV group and one in the control group withdrew because of time constraints (Figure 1). Of the 17 included in the WBV group, 16 completed $100 \%$ of the vibration training and one $70 \%$ of the training. All participants were instructed to maintain their normal diet, normal level of physical activity and prescribed medications throughout the experiment. The protocol of this study was approved by the Khon Kaen University Ethics Committee for Human Research (approval number HE521307) and Human Ethics Committee of Lincoln University (approval number 2009-52).

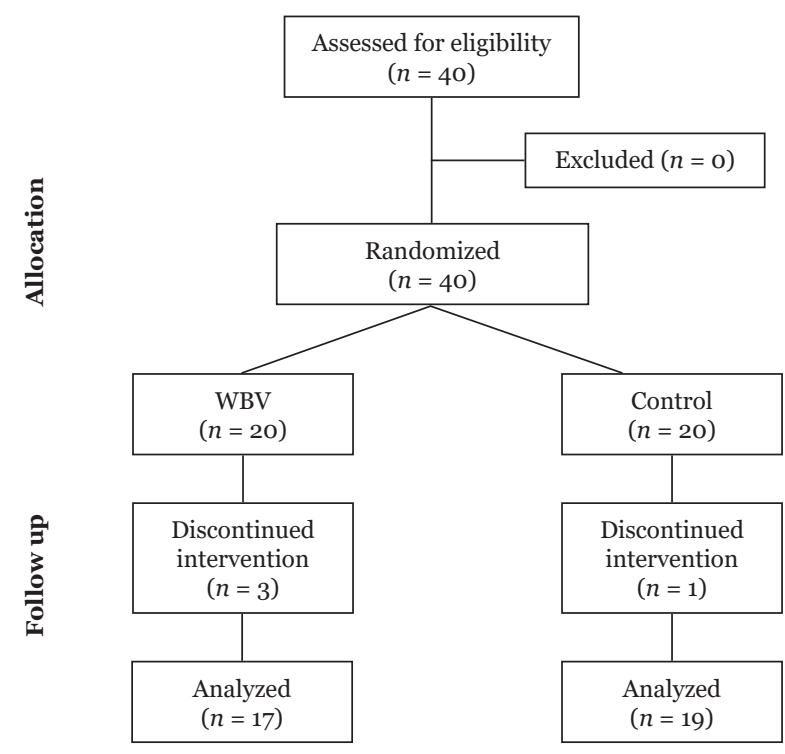

Figure 1. Consort diagram

\section{Whole Body Vibration Program}

The WBV group was trained on a vertical vibration machine (Fitvibe Excel; Belkin, Belgium) for two sets of 6 one-minute vibration squats interspersed with 20 second rest periods, 3 times per week for 12 weeks (total exercise time of 36 minutes per week). The six static positions consisted of, (a) a deep squat position (knee angle $90^{\circ}$ ), (b) high squat position (knee angle $125^{\circ}$ ), (c) high squat position (with raised heels), (d) slight knee flexion 1 (holding hand straps with shoulder flexion), (e) slight knee flexion 2 (holding hand straps with shoulder abduction) and (f) slight knee flexion 3 (holding hand straps with elbow flexion) (Figure 2). The training load increased progressively from an initial vibration frequency of $30 \mathrm{~Hz}$ and a platform amplitude shift of $2 \mathrm{~mm}$ (1.43 $\mathrm{g}$ in peak acceleration) in week 1 , to $40 \mathrm{~Hz}$, and $4 \mathrm{~mm}(7.34 \mathrm{~g}$ in peak acceleration) in week 5 , which was maintained until the end of the study (week 12). Adverse effects were recorded throughout the training program. The control group was told to maintain their normal physical activity levels for 12 weeks.

\section{Assessment}

\section{Resting Physiological Measures}

All physiological measures were taken at the clinic at the same time of day with participants wearing light clothing and no shoes. Weight and height were measured using a beam balance scale with an attached stadiometer (Detecto scales, 
New York, USA). Weight was measured by taking two measurements to the nearest $0.1 \mathrm{~kg}$. If the measures differed by more than $0.5 \mathrm{~kg}$, a third measurement was taken and the mean of the two closest measurements recorded. Height (to the nearest $0.1 \mathrm{~cm}$ ) was similarly determined. Blood pressure of the upper right arm was measured in sitting position using an automatic blood pressure monitor (Terumo ES H55, Terumo Co., Japan) after 15 minutes of rest.

\section{Blood Samples and Insulin Sensitivity}

All blood samples were taken by venipuncture of the antecubital vein in the morning after an overnight fast. The samples were analysed by experienced laboratory technicians using an automated machine (Cobas $^{\circledR} 6000$ analyser; Roche Diagnostics Corp., Indianapolis, IN, USA) for FBS, HbA1c, and an Automatic Gamma Counter (Wallac 1470 Wizard, Perkin Elmer Inc., Boston, MA, USA) for fasting insulin level. In this study, the quantitative insulin sensitivity check index (QUICKI) was calculated from the inverse of the sum of the logarithm of fasting insulin and fasting glucose $[1 /(\log$ (fasting insulin $\mu \mathrm{IU} / \mathrm{mL})$ $+\log$ (fasting glucose mg/dL)] (17).

\section{Ultrasound Doppler}

Duplex ultrasound (US) scanning of the right popliteal artery at the mid popliteal fossa with the patient in a prone position, after a 15 minute rest, was performed by an experienced radiologist. Each artery segment was examined with a $10-\mathrm{MHz}$ linear array transducer with a Toshiba Xario XG scanner (Toshiba Medical Systems, Tokyo, Japan) at a room temperature of $25{ }^{\circ} \mathrm{C}$. The color-flow mode was used to identify the vessel and to position the sample volume. The diameter of the arteries was obtained by longitudinal imaging, placing the tracker ball-guided calipers across the intimalluminal interphases of the near and far walls. Measurements were repeated three times, then averaged. A computer spectral analysis of the Doppler signals was completed to obtain profiles of the velocity waveforms (peak systolic velocity (PSV), end diastolic velocity (EDV)). This is possible by allowing the sample volume gate's to be adjusted to encompass the entire lumen of the artery and the angle of insonation being maintained at $60^{\circ}$. Doppler spectral waveforms containing aliasing, (noise due to venous flow or wall motion) were discarded. The mean of three different sets of flow estimations was obtained per patient both pre and post intervention.

\section{Statistical Analysis}

The data analysis was conducted as a per protocol analysis that did not include any participants that dropped out of the study. The statistical analysis was performed by SPSS version 19. The normal distribution of the data was evaluated by Kolmogorov-Smirnov test.

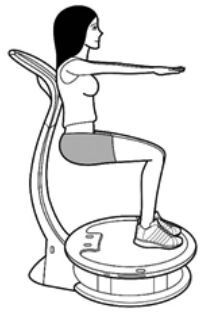

a. Deep squat position

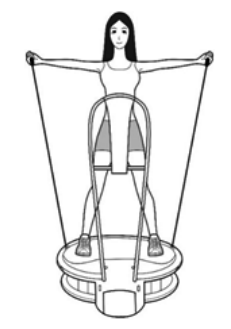

d. Slight knee flexion with shoulder abduction

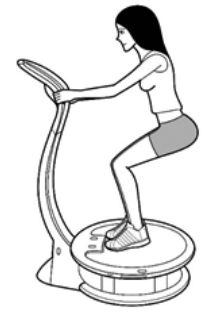

b. High squat position

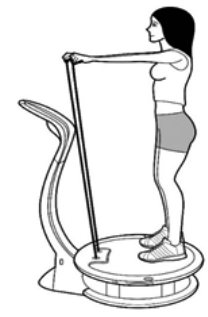

e. Slight knee flexion with shoulder flexion

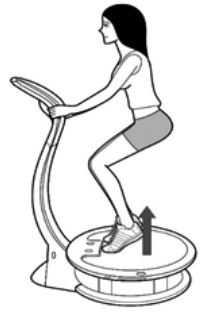

c. High squat position with raised heel

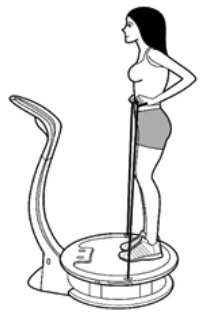

f. Slight knee flexion with elbow flexion

Figure 2. Positions of whole body vibration training 
The descriptive data is presented as mean, standard deviation and $95 \%$ confidence interval. The baseline characteristics of treatment and control groups were analysed by an independent sample $t$-test for continuous data and Chisquare test for nominal data. The analysis of pre- and post-intervention variables including body weight, body mass index (BMI), FBS, HbA1c, insulin level, insulin sensitivity, heart rate, systolic and diastolic blood pressure, peak systolic velocity, end diastolic velocity and vessel diameter was analysed using a paired sample $t$-test. Subsequently an analysis of covariance (ANCOVA), with adjusted mean change from baseline, was used to investigate differences between groups. Statistical significance was accepted at $P$-value $<0.05$.

\section{Results}

Thirty-six participants (13 males and 23 females, age 63.2 (7.7) years, BMI 26.1 (3.6) kg.m ${ }^{-2}$, FBS 142.8 (43.9) mg.dl ${ }^{-1}$, duration of diabetes $7.8(5.7)$ years, mean (SD)), completed the two testing protocols (baseline, post intervention). The per protocol analysis revealed there was no substantial difference between the WBV and control groups in age, duration of diabetes diagnosis, body weight, BMI, FBS, HbA1c (Table 1). No adverse effects of WBV were reported throughout the study.

After 12-weeks training, we found very little difference in body weight and BMI from baseline and between groups. Compared to baseline, fasting blood sugar and insulin sensitivity was substantially improved in both groups. Insulin levels significantly decreased in control group, but no change of insulin levels were observed in WBV group. However, the control group had significantly increased HbA1c by $0.4 \%$ (95\% CI: $0.1,0.7, P=0.009$ ) from baseline, while the participants who underwent vibration training had little change in Hb1Ac (-0.09\%, 95\%CI: $-1.0,0.8, p=0.819$ ). There was no statistically significant difference in FBS, HbA1c, insulin level and QUICKI between WBV and control group over the course of the study (Table 2).

The WBV group decreased resting diastolic blood pressure, whereas no substantial change was found in the control group during the study resulting in a substantial difference between groups over the study by $-7.1 \mathrm{mmHg}$ (95\% CI: $-10.9,-3.3, P=0.001$ ). We found very little difference in resting heart rate or resting systolic blood pressure within or between groups (Table 3). Ultrasound Doppler showed a decrease in peak systolic velocity by $-7.3 \mathrm{~cm} \cdot \mathrm{sec}^{-1}(95 \%$ CI: $-14.7,-0.03, P=0.049$ ) in the WBV group compared to control group. The end diastolic velocity in the WBV group decreased from baseline by $-3.1 \mathrm{~cm} \cdot \mathrm{sec}^{-1}$ (95\% CI: $-5.4,-0.8, P$ $=0.011)$, but there was no significant difference compared to control group. The diameter of the right popliteal artery in both groups did not differ throughout the study (Table 3).

\section{Discussion}

The main novel findings of this study are that 12 weeks of WBV training substantially

Table 1. Characteristics of WBV and control groups (mean (SD))

\begin{tabular}{|c|c|c|c|}
\hline & $\begin{array}{c}\text { WBV } \\
(n=17)\end{array}$ & $\begin{array}{c}\text { CON } \\
(n=19)\end{array}$ & $P$-value \\
\hline Age (year) & $60.9(11.2)$ & $63.9(4.9)$ & 0.318 \\
\hline Sex & $\mathrm{M} 7 / \mathrm{F} 10$ & M $6 /$ F 13 & \\
\hline Duration (year) of diabetes diagnosis & $6.9(5.4)$ & $8.5(5 \cdot 9)$ & 0.422 \\
\hline Body weight (kg) & $61.4(7.8)$ & $63.6(12.3)$ & 0.527 \\
\hline $\mathrm{BMI}\left(\mathrm{kg} \cdot \mathrm{m}^{-2}\right)$ & $25.7(2.4)$ & $26.7(4.2)$ & 0.406 \\
\hline FBS (mg.dl-1) & $148.7(38.0)$ & $130.1(25.5)$ & 0.100 \\
\hline HbA1c (\%) & $8.5(1.9)$ & $7.8(1.4)$ & 0.208 \\
\hline Insulin $\left(\mu \mathrm{IU} \cdot \mathrm{ml}^{-1}\right)$ & $20.8(6.7)$ & $22.6(10.6)$ & 0.539 \\
\hline QUICKI & $0.289(0.014)$ & $0.294(0.019)$ & 0.471 \\
\hline
\end{tabular}

BMI, body mass index; FBS, fasting blood sugar; HbA1c, glycosylated hemoglobin; QUICKI, the quantitative insulin sensitivity check index; CON, control; WBV, whole body vibration 
Table 2. Pre, post intervention and adjusted mean difference in BW, BMI, FBS, HbA1c and QUICKI of WBV and control groups (mean (SD))

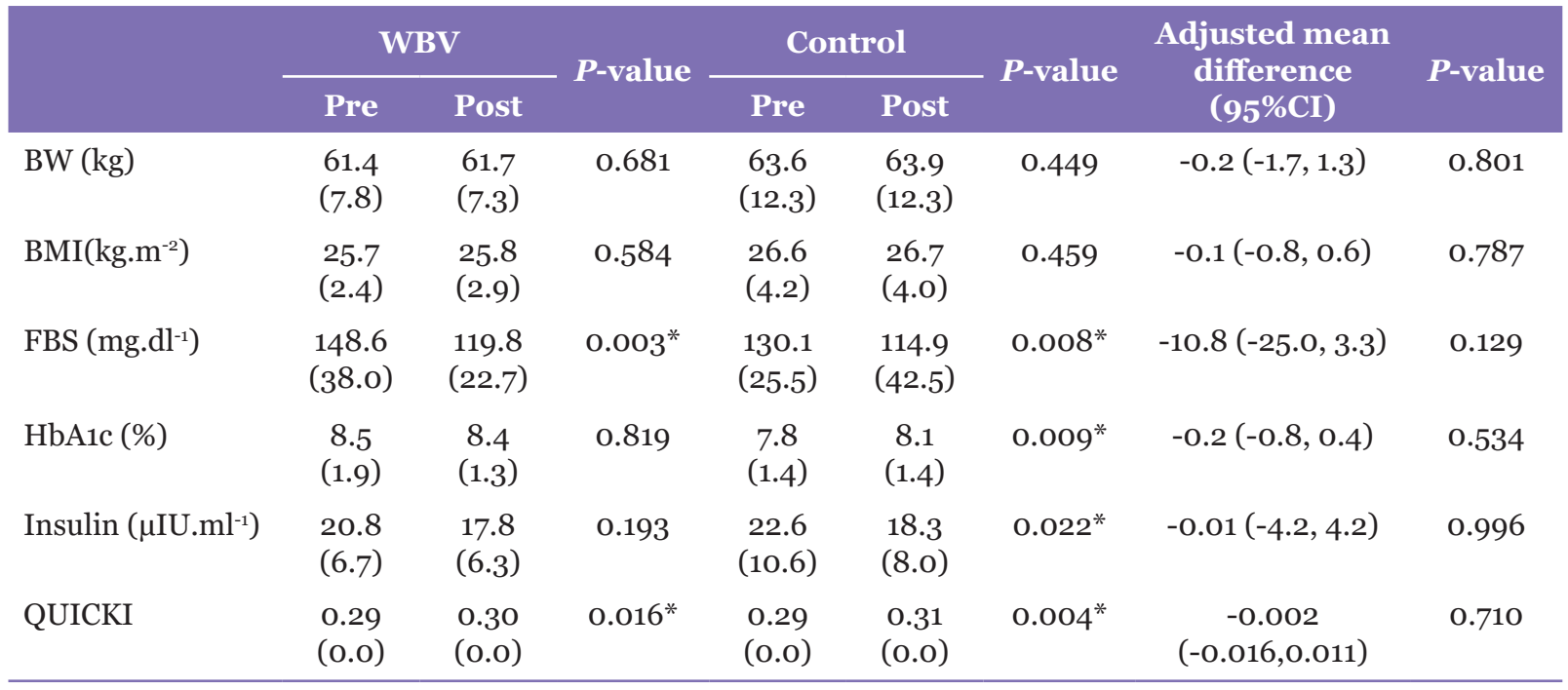

BW, body weight; BMI, body mass index; HR, heart rate; SBP, systolic blood pressure; DBP, diastolic blood pressure; FBS, fasting blood sugar; HbA1c, glycosylated hemoglobin; QUICKI, the quantitative insulin sensitivity check index; WBV, whole body vibration;

* significant differences between pre and post-test $(P<0.05)$.

Table 3. Pre, post intervention and adjusted mean difference in HR, SBP, DBP, PSV, EDV, vessel diameter of WBV and control groups (mean (SD))

\begin{tabular}{|c|c|c|c|c|c|c|c|c|}
\hline & \multicolumn{2}{|c|}{ WBV } & \multirow{2}{*}{$P$-value } & \multicolumn{2}{|c|}{ Control } & \multirow{2}{*}{$P$-value } & \multirow{2}{*}{$\begin{array}{l}\text { Adjusted mean } \\
\text { difference } \\
(95 \% \mathrm{CI})\end{array}$} & \multirow{2}{*}{$P$-value } \\
\hline & Pre & Post & & Pre & Post & & & \\
\hline HR (beat.min ${ }^{-1}$ ) & $\begin{array}{c}78.5 \\
(14.0)\end{array}$ & $\begin{array}{c}79.3 \\
(13.0)\end{array}$ & 0.680 & $\begin{array}{c}77.4 \\
(11.8)\end{array}$ & $\begin{array}{c}74.6 \\
(10.0)\end{array}$ & 0.238 & $4.0(-1.4,9.5)$ & 0.142 \\
\hline SBP (mmHg) & $\begin{array}{l}133.6 \\
(16.7)\end{array}$ & $\begin{array}{l}128.0 \\
(13.9)\end{array}$ & 0.145 & $\begin{array}{l}133.8 \\
(12.2)\end{array}$ & $\begin{array}{l}129.5 \\
(11.8)\end{array}$ & 0.073 & $-1.4(-8.7,5.9)$ & 0.698 \\
\hline DBP (mmHg) & $\begin{array}{c}74.6 \\
(15.6)\end{array}$ & $\begin{array}{c}66.1 \\
(5.9)\end{array}$ & $0.009^{*}$ & $\begin{array}{c}71.6 \\
(12.2)\end{array}$ & $\begin{array}{c}71.7 \\
(10.7)\end{array}$ & 0.951 & $-7.1(-10.9,-3.3)$ & $0.001^{* *}$ \\
\hline $\operatorname{PSV}\left(\mathrm{cm} \cdot \mathrm{sec}^{-1}\right)$ & $\begin{array}{c}55.6 \\
(14.6)\end{array}$ & $\begin{array}{c}51.6 \\
(11.3)\end{array}$ & 0.106 & $\begin{array}{c}63.6 \\
(19.4)\end{array}$ & $\begin{array}{c}63.8 \\
(17.2)\end{array}$ & 0.880 & $-7 \cdot 3(-14 \cdot 7,-0.03)$ & $0.049^{* *}$ \\
\hline $\operatorname{EDV}\left(\mathrm{cm} \cdot \mathrm{sec}^{-1}\right)$ & $\begin{array}{c}4.4 \\
(5.4)\end{array}$ & $\begin{array}{c}1.2 \\
(4.8)\end{array}$ & $0.011^{*}$ & $\begin{array}{c}5.3 \\
(6.8)\end{array}$ & $\begin{array}{c}3.5 \\
(4.2)\end{array}$ & 0.353 & $-2.0(-4.9,0.8)$ & 0.158 \\
\hline Diameter (mm) & $\begin{array}{c}6.1 \\
(0.9)\end{array}$ & $\begin{array}{c}6.2 \\
(0.5)\end{array}$ & 0.738 & $\begin{array}{c}6.2 \\
(0.8)\end{array}$ & $\begin{array}{c}6.2 \\
(0.8)\end{array}$ & 0.883 & $0.005(-0.3,0.4)$ & 0.976 \\
\hline
\end{tabular}

WBV, whole body vibration; HR, heart rate; SBP, systolic blood pressure; DBP, diastolic blood pressure; PSV, peak systolic velocity; EDV, end diastolic velocity; Diameter, popliteal artery diameter; * significant differences between pre and post-test $(P<0.05)$; ** significant differences between WBV and control group $(P<0.05)$.

reduced diastolic blood pressure and peak systolic velocity. However, the beneficial effects of WBV on glycaemic indices remained unclear.

It is thought that WBV stimulates the stretch reflex by activating muscle spindles to enhance muscle contraction (5), leading to increase glucose utilisation. The physiological mechanisms behind WBV's effect on glucose uptake of muscle cells is probably similar to physical exercise. These mechanisms include enhanced glucose transporter protein GLUT4 via activation of post-receptor insulin signalling and AMPK (AMP-activated protein kinase), increased cytoplasmic calcium, enhanced autocrine or paracrine (nitric oxide) response, and increased glycogen synthase and phosphorylase activities 
(18). Exercise also increases muscle glucose delivery and utilisation by increasing muscle blood flow, muscle capillary density and muscle mass $(19,20)$. In diabetic patients, WBV may help diabetic control by improving beta cell function and insulin resistance or enhanced responsiveness of muscle glucose uptake, similar to physical exercise $(7,21)$. The participants in both groups had lower FBS and insulin levels and higher insulin sensitivity in the post compared to the pre-test. These results may be explained by the fact that all participants received conventional treatment for their diabetes during the study including diet and medication control. While there was some within group changes as a result of the study, the between group results suggest that compared to controls using WBV training has little beneficial effect on indicators of glycaemic control (Table 2). Our results corroborate previous work by Baum et al. (2) and Behboudi et al. (8) who found that FBS and HbA1c were not significantly improved in the WBV group compared to control groups.

Perhaps the workload of traditional WBV training is not sufficiently intense or long enough to provide substantial change. An increased duration of training (per session), regularity of training (per week) and intensity of WBV such as adding loads on the body or dynamic exercise training while completing WBV may provide better results than reported here. In contrast, del Pozo-Cruz et al. (9) recently revealed WBV had significant beneficial effects on FBS and HbA1c. The baseline HbA1c of our study (8.5\% for intervention and $7.8 \%$ for control) is higher than del Pozo-Cruz et al. study (7.3\% for intervention and $7.0 \%$ for control) and we suspect the severity of diabetes may affect resultant glycemic outcomes. Future research should investigate to what extent severity of diabetes has on glycemic outcomes in a training study.

After 12-weeks of WBV training, both systolic and diastolic resting blood pressure in our subjects (substantial decrease in diastolic only) decreased compared to controls. It is suggested that WBV may dilate small blood vessels in muscles and thereby decrease peripheral vascular resistance (10). If this is the case, diastolic blood pressure, which is mainly determined by peripheral vascular resistance, would tend to decrease also. Since systolic blood pressure is mainly determined by cardiac output, changes in peripheral resistance would not be as obvious on systolic blood pressure. Similar to previous research, we found that WBV had little effect on resting heart rate (14). An interesting finding from the current study was that regular WBV training substantially reduced peak systolic velocity of the right popliteal artery. Whole body vibration may reduce vascular stenosis or resistance, which is positively correlated to peak systolic velocity (22). The proposed underlying mechanism suggests that acute vibration exposure may reduce peripheral vascular resistance by inhibiting vasoconstrictor substance release (endothelin) from smooth muscles (23). As with the adaptation process in exercise training, regular WBV training may alter peripheral blood vessels by increasing the size of the capillary bed of the skeletal muscle (24), or by increasing arteriole vasodilatation by releasing endothelial nitric oxide and prostaglandins (25).

We did not observe any adverse effects of WBV on type II diabetic patients such as pain, abnormal sensation, injury or hypoglycaemia. However, adverse effects related to diabetic complications such as retinopathy and nephropathy can occur with exercise. Vigorous exercise may trigger vitreous haemorrhage or retinal detachment in severe diabetic retinopathy, and may increase urinary protein excretion (26). Therefore, WBV with high loads and prolonged exposure times may put such patients at risk of these complications. This study did not perform retinal examination or monitor renal function, however, we suggest future research into the area of $\mathrm{WBV}$ and diabetes should consider such monitoring.

A limitation of this study is that the participants were diabetic patients who received diet control programs or oral medication and were trained at a primary care unit that allowed the participants to maintain their normal daily lifestyle; therefore, the results of this study cannot be applied to all diabetic patients in general. In addition, the variation in the glycemic measures was higher than expected which resulted in a reduction in the statistical power of the study for these glycemic measures. We would recommend future studies to stratify diabetic patients based on the degree of severity of diabetes using baseline HbA1c or duration of disease measures.

\section{Conclusion}

Our study revealed WBV training in type II diabetic patients had no significant beneficial effects on the control of FBS, HbA1c, insulin 
levels and insulin sensitivity compared to a control group; however, vibration training reduced resting diastolic pressure and peak systolic velocity in these type II diabetic patients.

\section{Acknowledgements}

Special thanks to all the patients for their contribution and participation, Ms Pornsawan Wongkarnchanakul and Lee Wattanan for supporting the Fitvibe $^{\circledR}$ whole body vibration machine. Thanks also to Dr Kaewjai Thepsuthammarat, Khon Kaen University for the statistical analysis.

\section{Conflicts of Interest}

None

\section{Funds}

This study was supported by Post-Graduated Lincoln University Research Funding New Zealand (ID 1081357).

\section{Authors' Contributions}

Conception and design: NM, AM, WP, MJH

Analysis and interpretation of the data: NM, AM, WP, $\mathrm{MJH}$

Drafting of the article: NM, AM, WP

Critical revision of the article for important intellectual content: $\mathrm{MJH}$

Final approval of the article: NM, AM, WP, MJH

Provision of study materials: NM

Statistical expertise: NM, MJH

Obtaining funding: NM

Administrative, technical, or logistic support: NM,

$\mathrm{MJH}$

Collection and assembly of data: NM, AM, WP

\section{Correspondence}

Dr Nuttaset Manimmanakorn

MD (Khon Kaen University), PhD (Lincoln University)

Department of Physical Medicine and Rehabilitation,

Faculty of Medicine, Khon Kaen University,

Khon Kaen, 40002, Thailand.

Telephone and Fax: +66 43348392

E-mail: natman@kku.ac.th

\section{References}

1. American Diabetes Association. Diagnosis and classification of diabetes mellitus. Diabetes care. 2017;40(Suppl 1):S11-S24. https://doi. org/10.2337/dc17-Soo5

2. Baum K, Votteler T, Schiab J. Efficiency of vibration exercise for glycemic control in type 2 diabetes patients. Int J Med Sci. 2007;4(3):159163. https://doi.org/10.7150/ijms.4.159

3. Prisby RD, Lafage-Proust $\mathrm{MH}$, Malaval L, Belli A, Vico L. Effects of whole body vibration on the skeleton and other organ systems in man and animal models: what we know and what we need to know. Ageing Res Rev. 2008;7(4):319-329. https://doi.org/10.1016/j.arr.2008.07.004

4. Raulino RS, Aguiar FM, Avelar NC, Costa IG, Soares JD, Lacerda AC. Energy expenditure and substrate utilization during whole body vibration. Rev Bras Med Esporte. 2015;21(2):122126. http://dx.doi.org/10.1590/151786922015210201976

5. Cardinale M, Bosco C. The use of vibration as an exercise intervention. Exerc Sport Sci Rev. 2003;31(1):3-7. https://doi. org/10.1097/00003677-200301000-00002

6. Bosco C, Iacovelli M, Tsarpela O, Cardinale M, Bonifazi M, Tihanyi $\mathrm{J}$ et al. Hormonal responses to whole-body vibration in men. Eur $J$ Appl Physiol. 2000;81(6):449-454. https://doi. org/10.1007/s004210050067

7. Holloszy JO. Exercise-induced increase in muscle insulin sensitivity. $J$ Appl Physiol. 2005;99(1):338-343. https://doi.org/10.1152/ japplphysiol.o0123.2005

8. Behboudi L, Azarbayjani MA, Aghaalinejad $H$, Salavati M. Effects of aerobic exercise and whole body vibration on glycaemia control in type 2 diabetic males. Asian $J$ Sports Med. 2011;2(2):83-90.

9. del Pozo-Cruz B, Alfonso-Rosa RM, del Pozo-Cruz J, Sañudo B, Rogers ME. Effects of a 12-wk wholebody vibration based intervention to improve type 2 diabetes. Maturitas. 2014;77(1):52-58. https:// doi.org/10.1016/j.maturitas.2013.09.005 
10. Kerschan-Schindl K, Grampp S, Henk C, Resch H, Preisinger E, Fialka-Moser V, et al. Whole-body vibration exercise leads to alterations in muscle blood volume. Clin Physiol. 2001;21(3):377382. https://doi.org/10.1046/j.13652281.2001.00335.x

11. Lohman EB 3rd, Petrofsky JS, Maloney-Hinds C, Betts-Schwab H, Thorpe D. The effect of whole body vibration on lower extremity skin blood flow in normal subjects. Med Sci Monit. 2007;13(2):CR71-76.

12. Lythgo N, Eser P, De Groot P, Galea M. Wholebody vibration dosage alters leg blood flow. Clin Physiol Funct Imaging. 2009;29(1):53-59. https://doi.org/10.1111/j.1475-097X.2008.00834.x

13. Button C, Anderson N, Bradford C, Cotter JD, Ainslie PN. The effect of multidirectional mechanical vibration on peripheral circulation of humans. Clin Physiol Funct Imaging. 2007;27(4):211-216. https://doi.org/10.1111/ j.1475-097X.2007.00739.x

14. Hazell TJ, Thomas GW, Deguire JR, Lemon PW. Vertical whole-body vibration does not increase cardiovascular stress to static semi-squat exercise. Eur J Appl Physiol. 2008;104(5):903-908. https://doi.org/10.1007/s00421-008-0847-y

15. Johnson PK, Feland JB, Johnson AW, Mack GW, Mitchell UH. Effect of whole body vibration on skin blood flow and nitric oxide production. J Diabetes Sci Technol. 2014;8(4):889-894.

16. Sañudo B, Alfonso-Rosa R, del Pozo-Cruz B, del Pozo-Cruz J, Galiano D, Figueroa A. Whole body vibration training improves leg blood flow and adiposity in patients with type 2 diabetes mellitus. Eur J Appl Physiol. 2013;113(9):2245-2252. https://doi.org/10.1007/s00421-013-2654-3

17. Katz A, Nambi SS, Mather K, Baron AD, Follmann DA, Sullivan G, et al. Quantitative insulin sensitivity check index: a simple, accurate method for assessing insulin sensitivity in humans. $J$ Clin Endocrinol Metab. 2000;85(7):2402-2410. doi: 10.1210/jcem.85.7.6661

18. Pereira LO, Lancha AH, Jr. Effect of insulin and contraction up on glucose transport in skeletal muscle. Prog Biophys Mol Biol. 2004;84(1):127. http://dx.doi.org/10.1016/Soo79-6107(03) 00055-5
19. Colberg SR, Sigal RJ, Fernhall B, Regensteiner JG, Blissmer BJ, Rubin RR, et al. Exercise and type 2 diabetes: the American College of Sports Medicine and the American Diabetes Association: joint position statement. Diabetes Care. 2010;33(12):e147-e167. https://doi. org/10.2337/dc10-9990

20. Laughlin MH, Roseguini B. Mechanisms for exercise training-induced increases in skeletal muscle blood flow capacity: differences with interval sprint training versus aerobic endurance training. J Physiol Pharmacol. 2008;59(Suppl 7):71-88.

21. Bloem CJ, Chang AM. Short-term exercise improves beta-cell function and insulin resistance in older people with impaired glucose tolerance. $J$ Clin Endocrinol Metab. 2008;93(2):387-392. https://doi.org/10.1210/jc.2007-1734

22. Mofidi R, Powell TI, Crotty TB, Sheehan SJ, Mehigan D, MacErlaine DP, et al. Increased internal carotid artery peak systolic velocity is associated with presence of significant atherosclerotic plaque instability independent of degree of ICA stenosis. Int $J$ Angiol. 2005;14(2):74-80. https://doi.org/10.1007/ s00547-005-1079-1

23. Nakamura H, Ariizumi M, Okazawa T, Nagase H, Yoshida M, Okada A. Involvement of endothelin in peripheral circulatory change induced by handarm vibration. Cent Eur J Public Health.1995;3 Suppl:27-30.

24. Gielen S, Schuler G, Adams V. Cardiovascular effects of exercise training. Circulation. 2010;122(12):1221-1238. https://doi.org/ 10.1161/CIRCULATIONAHA.110.939959

25. Whyte JJ, Laughlin MH. The effects of acute and chronic exercise on the vasculature. Acta physiologica (Oxford, England). 2010;199(4):441-450. https://doi.org/10.1111/ j.1748-1716.2010.02127.x

26. Sigal RJ, Kenny GP, Wasserman DH, CastanedaSceppa C, White RD. Physical activity/exercise and type 2 diabetes: a consensus statement from the American Diabetes Association. Diabetes care. 2006;29(6):1433-1438. https://doi. org/10.2337/dco6-9910 